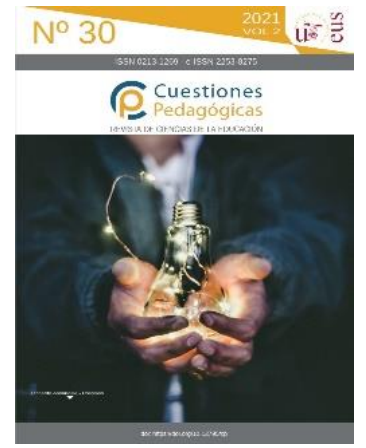

\title{
Los proyectos didácticos en la educación primaria indígena: una estrategia para una educación pertinente desde una perspectiva cultural y lingüística.
}

\author{
Didactic projects in indigenous primary education: a \\ strategy for relevant education from a \\ cultural and linguistic perspective.
}

Recibido: 06/03/2021 | Revisado: 09/03/2021 | Aceptado: 02/08/2021 | Online first: 01/09/2021 Publicado: 31/12/2021

\author{
Noel Palestino-de-Jesús \\ Universidad Autónoma de Tlaxcala (México) \\ noelpalestino@hotmail.com \\ https://orcid.org/0000-0002-7993-7378
}

Resumen: En este artículo se presentan los resultados de un estudio cualitativo acerca de la implementación de los Proyectos Didácticos (PD) como estrategia empleada por colectivos docentes de educación primaria indígena en el estado de Veracruz, México, para dotar de pertinencia cultural y lingüística a la educación que imparten. Se utilizó una metodología de carácter etnográfico, se realizaron entrevistas semiestructuradas para conocer la experiencia de los docentes y sus procedimientos empleados en el trabajo con esta herramienta pedagógica; por otra parte, la observación directa en las aulas de sesiones de trabajo sirvió para contrastar y complementar la información. Entre los resultados, se observó que para los maestros es prioritaria la valoración de la cultura indígena nahua, consideran, además, que existe una estrecha relación entre significatividad del aprendizaje y pertinencia cultural, por lo que al trabajar con los PD recurren a saberes tradicionales para contextualizar los contenidos del currículum nacional, y a la lengua indígena para movilizar los conocimientos suscitados,
Abstract: This article presents part of the results of a qualitative study about the implementation of Didactic Projects (DPs) as a strategy used by indigenous primary education teaching groups to provide cultural and linguistic relevance to the education in the state of Veracruz, México. We used ethnographic approach. On the one hand semi-structured interviews were carried out to know the experience of the teachers and their procedures used at work with this pedagogical tool. On the other hand, direct observation in the classroom of some work sessions served to contrast and complement the information obtained in the interviews. The results of the study show that the Nahua culture has become a priority for the indigenous teachers. They also consider that there in a close relationship between learning significance and cultural relevance. In this way, they resort to traditional knowledge gained, thus contributing to the revitalization of their native culture. Undoubtedly, the possibility of improving the relevance of the educational practices offered in the indigenous communities exists, so educational authorities must address the lack of teacher training and educational materials. 
contribuyendo con esto, a la revitalización de su cultura originaria. Sin duda, existe la posibilidad de mejorar la pertinencia en las prácticas educativas que se ofertan en las comunidades indígenas, por lo que las autoridades en la materia deben atender carencias en rubros como la capacitación docente y los materiales educativos

Palabras clave: Pertinencia de la educación, Estrategias educativas, Educación intercultural, Educación comunitaria, Conocimientos tradicionales.
Keywords: Educational relevance, Educational strategies, Intercultural education, Social education, Traditional knowledge

\section{Introducción}

Con el reconocimiento del Estado mexicano sobre su composición multicultural y plurilingüe en la última década del siglo $\mathrm{XX}$, los pueblos originarios lograron conquistar espacios de participación y decisión en el diseño curricular que demandaban desde años atrás (Muñoz, 1999), esto abrió paso para que la pertinencia educativa se convirtiera en un imperativo esencial para atender la diversidad cultural, y, además, aspirar a una educación de calidad (UNESCO, 2007), sobre todo, cuando diversos diagnósticos (SEP-DGEl, 1990; Jiménez y Mendoza, 2012; SEP-CGEIB, 2014; Niembro y Mendoza, 2017), señalan que el servicio educativo que recibe la población indígena en México es deficiente y carece de pertinencia cultural y lingüística.

En la II Reunión Intergubernamental del Proyecto Regional de Educación para América Latina y el Caribe (EPT/PRELAC), se concluyó para que la educación sea pertinente "tiene que ser flexible y adaptarse a las necesidades y características de los estudiantes y de los diversos contextos sociales y culturales" (UNESCO, 2007, p. 9).

La pertinencia debe ser un referente primordial en el diseño curricular si es que se espera que una educación desarrolle efectivamente las capacidades y potencialidades del alumnado (UNESCO, 2007), bajo este enfoque, el proceso educativo se articula y desarrolla en torno a los alumnos, los aprendizajes deben estar en función de sus intereses y necesidades para resultar significativos. Así mismo, el contexto cultural es un requisito fundamental para que los aprendizajes resulten valiosos, y crucial para dotar al aprendizaje de significatividad: "un aprendizaje pertinente es, pues aquel, que resulta culturalmente significativo para el sujeto que aprende" (Hevia, Hirmas y Peñafiel, 2002, p.33). 
Un currículum en educación básica diseñado para atender la diversidad y cumplir con la condición de una educación pertinente ha sido un avance significativo, no obstante, debe trabajarse en el logro de prácticas educativas basadas en la diversidad que doten a la educación de pertinencia y significatividad para los diferentes grupos culturales (UNESCO, 2007). Por ello, cobra relevancia el esfuerzo que realizan los docentes de educación indígena para ejercer prácticas pertinentes orientadas por el enfoque intercultural bilingüe.

Es importante que cada comunidad educativa participe activamente y con libertad, para definir lo que es educativamente pertinente a sus intereses y necesidades propias a su cultura, del mismo modo, para determinar las estrategias de acción con las que han de lograr sus propósitos. Las prácticas educativas con pertinencia cultural deben encargarse de reforzar la identidad a través de la identificación y priorización de los elementos significativos de cada cultura (Salazar; Saldívar; Limón; Estrada y Fernández, 2015).

Investigaciones educativas señalan la centralidad de la cultura y la lengua en el ejercicio de una educación pertinente, concluyen que estos dos elementos son primordiales para el sustento pedagógico de la acción docente (Niembro y Mendoza, 2017). En ese contexto, colectivos docentes de escuelas de la zona 832 de educación primaria indígena en estado de Veracruz, México, han implementado Proyectos Didácticos (PD) con elementos de la cultura nahua para dotar de pertinencia cultural a sus prácticas educativas, este artículo presenta la experiencia educativa, previa al confinamiento por el SARS-CoV-2, de dos escuelas en las que se trabaja con esa estrategia, gracias a la cual, han logrado vincular a sus alumnos no solo con el conocimiento escolar, sino también, con el de su realidad cultural. Durante el periodo en el que se realizó la investigación, en la ESC1 se desarrolló el proyecto "La celebración del día de muertos", mientras que en la ESC2 un proyecto de "Herbolaria".

\section{¿Qué son, en qué consisten, y cuál es la finalidad de los PD en la educación indígena?}

Los PD o también llamados escolares, son un recurso pedagógico que utilizan los docentes de esta modalidad educativa para dotar a su práctica educativa de pertinencia cultural y lingüística. Con esta estrategia, triangulan los contenidos educativos establecidos en el currículum nacional con saberes tradicionales de la cultura local, y, a su vez, con el uso de la lengua indígena. Como resultado, no sólo han mejorado sus procesos de enseñanza-aprendizaje, sino también, han impactado favorablemente en sus comunidades.

La modalidad del trabajo por proyectos didácticos se pensó para lograr operar una enseñanza situada que promueva la integración de la escuela a la comunidad donde habita. Un proyecto es un plan de trabajo libremente escogido con el objetivo de hacer algo que interesa, sea un problema que se quiere resolver o una tarea que hay que llevar a cabo (SEP, 2017, p. 41). 
Con los proyectos, directivos y docentes además de alcanzar los propósitos del programa escolar, buscan que los aprendizajes sean significativos para los alumnos además de revitalizar la cultura indígena. Se valen de saberes tradicionales para contextualizar los contenidos educativos del currículum nacional, mientras que la lengua indígena adquiere sentido para los alumnos al comunicar aprendizajes o situaciones propias de su cultura emanados de los proyectos, tanto en el ámbito escolar como en el comunitario, para la SEP (2017):

Estos proyectos integran los contenidos curriculares referidos a la tradición oral y a la tradición escrita de manera articulada; dan sentido al aprendizaje, favorecen el intercambio y la solidaridad entre iguales; y brindan la posibilidad de desarrollar la autonomía y la responsabilidad social de los educandos (p. 41).

Pedagógicamente, Arias (2017), señala que los PD "se sitúan dentro del marco filosófico constructivista e implican una estrategia metodológica que integra los procedimientos necesarios para desarrollar aprendizajes individuales o colectivos con base en actividades innovadoras" (p. 51). Por otro lado, Riess (2013), sostiene que como estrategia didáctica los PD se fundamentan en el ejercicio de la intraculturalidad, la cual "consiste en el reconocimiento, fortalecimiento, valoración y desarrollo de la propia cultura por sus miembros" (p. 20). Los directivos expresan que los resultados no sólo deben percibirse en el ámbito escolar, sino también en el comunitario.

Para estructurar el PD, los docentes de cada escuela elaboran de manera colectiva y democrática un plan general en el que definen el tema del proyecto, los objetivos, las estrategias didácticas, las actividades, los responsables y los recursos a utilizar. El proyecto se desarrolla en un lapso de tres meses, durante los cuales, los colectivos se reúnen semanalmente para informar los avances, las complicaciones, y para realizar los reajustes necesarios. Se requiere que los docentes conozcan sobre el tema principal, el cual es el eje articulador de los demás elementos considerados en el proyecto, esto los ha llevado a realizar investigación documental y de campo con diferentes personas de las comunidades: ancian@s con el reconocimiento social y comunitario por sus conocimientos, curander@s, yerber@s, y demás personas con diversos oficios.

\section{Metodología}

Debido a la naturaleza cultural del fenómeno estudiado, se optó por una metodología de tipo etnográfico. De acuerdo con Vásquez y Valenzuela (2013), la etnografía no es sólo un método, es un contenido técnico ligado al trabajo en campo, un momento de observación de la reflexión antropológica, un trabajo global que involucra técnicas que van de la observación participante a las entrevistas. Así que para explorar y conocer la realidad que experimentan las comunidades educativas con la forma de trabajo de 
los PD, se optó por este método, el cual, se caracteriza por la generación de datos a partir de la cotidianidad de la realidad de los miembros de un grupo particular, en un proceso en el cual, se mira hacia adentro para traducir hacia afuera (Dietz, 2017).

\section{Contextualización}

\section{Las escuelas}

La investigación se efectúo en dos escuelas primarias indígenas multigrado ubicadas en comunidades del municipio nahua-hablante de San Andrés Tenejapan, Veracruz, México. La escuela uno (ESC1) es pentadocente, su plantilla la constituyen tres maestros y dos maestras. Por su parte, la escuela dos (ESC2) es tridocente con dos maestros y una maestra, en ambas instituciones los directivos atienden grupo. En el ciclo escolar 2019-2020 la matrícula de la ESC1 fue de 88 alumnos, de los cuales 37 son hablantes de lengua indígena $(\mathrm{HLI})$, mientras que la matrícula de la ESC2 fue de 65 alumnos, 19 de ellos son HLI.

\section{El municipio}

San Andrés Tenejapan se localiza en la zona montañosa del centro del estado de Veracruz, México, el $88 \%$ de su extensión es boscosa y solo el $11 \%$ del territorio se utiliza para la agricultura, ya que el suelo no tiene uso potencial para esta actividad económica. Esto ha hecho que la población de las comunidades se dedique a actividades económicas primarias como la extracción de madera, cría de animales de corral, agricultura en la modalidad de subsistencia, y en la elaboración y comercialización de productos derivados del maíz (SEFIPLAN, 2018).

Es uno de los municipios más pequeños entidad veracruzana ya que ocupa sólo el $0.03 \%$ de su superficie total. La cabecera municipal y las localidades cuentan con servicios de luz eléctrica, agua potable y transporte público que las comunica con los municipios vecinos y con la urbe más próxima que es la ciudad de Orizaba. La comunicación entre comunidades es a través de caminos secundarios y en la región no hay servicio telefónico ni de internet. Por otro lado, está considerado con alto grado de marginación, y por su índice de rezago, ocupa a nivel nacional el lugar 493 de aproximadamente 2460 municipios en total (CONEVAL, 2015).

Hasta el 2015, el municipio contaba con 2,906 habitantes, con un porcentaje de población de $78.1 \%$ de población en situación de pobreza, del cual, el $51.3 \%$ se considera en pobreza moderada y el $26.8 \%$ en pobreza extrema. En el ámbito educativo, el $42 \%$ de la población se registró con rezago educativo; el 17\% de la población de 15 años o más es analfabeta; el 3\% de población de 6 a 14 años no asiste a la escuela, y el $69 \%$ de la población con 15 años o más no completó su educación básica (CONEVAL, 2015).

Los idiomas que se hablan son el español y el náhuatl de la región de las altas montañas del estado de Veracruz. Hasta el 2018, el $82.77 \%$ de la población total del 
municipio era hablante de lengua indígena, mientras que el porcentaje de su población indígena que no habla español es del 1.11\% (SEFIPLAN, 2018). El náhuatl se considera una lengua mayor autónoma a la que se le asigna una vitalidad e historicidad. De acuerdo a su función, es una lengua de grupo que generalmente se utiliza como lengua en la religión y en la educación (Petrovic, 2017).

La palabra náhuatl tiene varios significados, entre ellos están: "el que habla bonito", "el que habla claro", "el que habla bien", popularmente, el idioma también es llamado nahua o mexicano. Antes de la conquista española, este idioma se hablaba en gran parte de lo que ahora es México, extendiéndose hasta regiones de Guatemala, el Salvador y Nicaragua (AVELI, 2010)

Otro rasgo cultural importante de esta región nahua es que la población manifiesta una cosmovisión particular del mundo expresada en el respeto que tienen por la tierra y en sus formas de organización social y política. Las personas mantienen una relación espiritual con la naturaleza, o, con la "Madre Tierra", desde épocas ancestrales. También conservan relaciones sociales de tipo comunitario que se caracterizan por la solidaridad y la cooperación, por ejemplo, esto se observa en la organización del trabajo por faenas o trabajo comunitario colectivo para realizar obras de construcción o mantenimiento en las comunidades.

\section{La entrevista semiestructurada}

Se construyó en dos etapas, en la primera se elaboró un guion con preguntas abiertas relacionadas al tema de las prácticas educativas pertinentes y se piloteó con un par de docentes. Este ejercicio permitió, en una segunda etapa, definir los tópicos y las preguntas definitivas a partir de un proceso de codificación y categorización de la información aportada por los primeros entrevistados. Las preguntas finales se derivaron de categorías como "prácticas educativas pertinentes", "proyectos didácticos", "planeación y ejecución", "contenidos", "competencias interculturales", "materiales educativos" y "evaluación".

\section{La observación directa}

Lo recabado con esta técnica permitió llevar a cabo un proceso de complementación, diversificación y contrastación de la información para enriquecer los resultados de la investigación, al igual que las entrevistas, estuvo orientada por un guion elaborado con base en las categorías ya mencionadas. La forma manifiesta en la que se llevó a efecto no interfirió con el desarrollo natural de las actividades ni en el comportamiento de docentes y alumnos, esto se debió a que previamente realicé varias visitas a las escuelas durante la fase del diseño y realización de las entrevistas, así que mi presencia llegó a resultar familiar para los actores.

\section{Participantes}

Se trabajó con cuatro docentes, dos por cada escuela, para efecto de identificación se abrevian DOC1, DOC2, DOC3 y DOC4, todos ellos declaran ser miembros de la cultura nahua, que su lengua materna es el náhuatl y su segunda 
lengua el español, tres de ellos radican en municipios aledaños y una maestra es nativa de la misma comunidad en la que trabaja. Los cuatro cuentan con el nivel de licenciatura, aunque tres docentes iniciaron a laborar solo con estudios de bachillerato, cubriendo el perfil profesional ya estando en servicio. Todos cuentan con una antigüedad en el empleo mayor a los quince años, mientras que en sus actuales centros de trabajo tienen laborando dos, nueve, diez y dieciséis años respectivamente.

\section{Proceso de análisis}

En la investigación etnográfica, con la transcripción y protocolización de lo recabado mediante los diferentes instrumentos se articula la fase de construcción de los datos, con la fase de análisis e interpretación de la información (Dietz, 2017).

De tal manera, que, una vez transcrita la información recabada mediante las entrevistas y la observación directa en cada uno de los protocolos, se efectuó el análisis e interpretación. Siguiendo a Dietz (2017), se combinaron dos niveles de análisis: uno de tipo vertical, protocolo por protocolo, y otro de tipo horizontal, entre protocolos. El primero condensa los datos construidos en cada uno de los entrevistados, mientras que el segundo permite contrastar diferentes puntos de vista sobre una misma cuestión.

Posteriormente se efectuó un proceso de codificación y categorización definitiva para realizar un análisis hermenéutico asistido por el software de Atlas.ti, el cual contribuyó a descubrir y ordenar ideas, pensamientos y significados emanados tanto de la perspectiva de los sujetos (perspectiva emic), como de mi interpretación (perspectiva etic). Finalmente, la redacción de los resultados se realizó con base en cada categoría, las cuales se convirtieron en los distintos apartados que a continuación se presentan.

\section{Resultados y discusión}

\section{Planeación y ejecución de las actividades}

Para iniciar a trabajar el proyecto de "La celebración del día de muertos", el encargado de tercer y cuarto grado en la ESC1 me expuso su planeación (ver tabla 1) para las tres primeras sesiones.

Previamente acordado me presenté a la primera sesión, para los alumnos no fue sorpresa ya que anteriormente había asistido a la escuela en varias ocasiones para entrevistarme con el director y los docentes, incluido su profesor, la sesión inició de esta manera:

DOC4: A ver, quiero que se sienten en su lugar y que me pongan atención, quiero informarles que vamos a estar trabajando con un proyecto muy bonito, es una tradición nuestra muy antigua y se trata de recibir a aquellas personas que ya no están con nosotros. 
Sergio: ¡Que ya se murieron!

DOC4: Sí, pero siguen vivas en nuestra mente y en nuestros corazones.

Rogelio: Se fueron al cielo y desde allá nos están viendo.

José: Sí, dice mi mamá que están vivos solo que ya no los vemos, pero nos vienen a visitar.

Soledad: ¡Maestro!.. Mi abuelito se murió el otro día, pero dice mi abuelita que ahorita que sea el día de muertos va a venir a vernos.

DOC4: Bueno, muy bien, nosotros recordamos a nuestros difuntos con la tradición del día de muertos, o también le llaman de los fieles difuntos, ¿han oído hablar de ella?

Alumnos en coro: ¡Sí!

DOC4: ¿Con qué otro nombre la conocen?:

Jimena: ¡Los muertitos!

Josué: ¡El día de todos santos!

DOC4: ¿Y saben cómo se le llama en náhuatl a esta tradición?

Josué: ¡Omiki!

DOC4: ¿Así se le dice?

Carmen: Eso lo dicen cuando se mueren, pero es para los animales.

José: ¡Si es cierto! Cuando se muere un perro o un marrano le dicen: ya omiki, pero con la gente dicen opiliwitl, que ya está muerto.

Jimena: ¡Sí, opiliwitl!,

Después de este intercambio entre los alumnos, el maestro explicó que ambas expresiones significaban "ya murió" o "ya se murió", también mencionó que la diferenciación de los términos aplicados a personas o animales era correcta. Acto seguido les dijo a los alumnos que la celebración del día de muertos en náhuatl es conocida como: mikkaiwitl, confirmó con ellos la palabra preguntándoles ¿cómo se dice?, y los alumnos respondieron en coro: ¡mikkaiwitt!

El maestro lo anotó en el pizarrón junto a la oración "La celebración del día de muertos" y les pidió a sus alumnos que lo escribieran en su cuaderno. Posteriormente, el profesor les dijo que realizarían una investigación sobre el altar de muertos, que debían preguntar a sus padres, tíos, abuelos o vecinos si era necesario, qué elementos se colocaban en la ofrenda, cuál era su significado y cómo se armaba el altar.

Mientras el maestro explicaba lo que los alumnos debían investigar también lo escribía en el pizarrón, para armar los equipos primero les dio la opción a sus alumnos 
de elegir en cual querían estar, finalmente, a los alumnos que no eligieron los asignó de manera que los equipos quedaran equilibrados en cuanto al número de integrantes (Diario de campo, ESC1, 2019).

\section{Tabla 1}

Planeación del DOC4. Segundo ciclo, tercer y cuarto grado.

\begin{tabular}{|c|c|c|c|}
\hline \multicolumn{4}{|c|}{$\begin{array}{l}\text { Propósito: Que los alumnos conozcan la constitución y el significado del altar de muertos a través } \\
\text { de la investigación con familiares y miembros de la comunidad. }\end{array}$} \\
\hline $\begin{array}{l}\text { Prácticas sociales } \\
\text { de lenguaje }\end{array}$ & $\begin{array}{l}\text { Aprendizajes } \\
\text { esperados }\end{array}$ & Actividades & Recursos \\
\hline $\begin{array}{l}\text { Registrar y difundir } \\
\text { las costumbres y } \\
\text { tradiciones }\end{array}$ & $\begin{array}{l}\text { Recopila } \\
\text { información de } \\
\text { diversas fuentes } \\
\text { (orales o escritas) } \\
\text { para preparar una } \\
\text { exposición. }\end{array}$ & $\begin{array}{l}\text { 1.- investigar el nombre de los } \\
\text { elementos del altar en náhuatl } \\
\text { y en español. } \\
\text { 2.- Investigar el significado de } \\
\text { los elementos en el altar. } \\
\text { 3.- Investigar cómo se arma un } \\
\text { altar de muertos. }\end{array}$ & $\begin{array}{l}\text { Libreta, lápiz, } \\
\text { libros de la } \\
\text { biblioteca. }\end{array}$ \\
\hline $\begin{array}{l}\text { Elaboración de } \\
\text { textos que presentan } \\
\text { información } \\
\text { resumida } \\
\text { proveniente de } \\
\text { diversas fuentes. }\end{array}$ & $\begin{array}{l}\text { Elabora resúmenes } \\
\text { en los que se } \\
\text { describen procesos } \\
\text { naturales y } \\
\text { acontecimientos } \\
\text { históricos. }\end{array}$ & $\begin{array}{l}\text { En trabajo por equipos los } \\
\text { alumnos organizan la } \\
\text { información por temas y } \\
\text { elaboran resúmenes. }\end{array}$ & $\begin{array}{l}\text { Libreta, } \\
\text { lapiceros, lápiz, } \\
\text { pinturas, } \\
\text { fotocopias. }\end{array}$ \\
\hline $\begin{array}{l}\text { Intercambio oral de } \\
\text { experiencias y } \\
\text { nuevos } \\
\text { conocimientos. }\end{array}$ & $\begin{array}{l}\text { Presenta } \\
\text { exposiciones } \\
\text { acerca de las } \\
\text { características } \\
\text { físicas de su } \\
\text { localidad y de } \\
\text { algunos } \\
\text { acontecimientos } \\
\text { históricos de esta. }\end{array}$ & $\begin{array}{l}\text { Realizarán una exposición } \\
\text { sobre el tema que trabajaron } \\
\text { (en el caso de los alumnos } \\
\text { que saben hablarlo podrán } \\
\text { hacerlo en náhuatl). Se } \\
\text { apoyarán con la elaboración } \\
\text { de carteles donde utilicen } \\
\text { palabras en náhuatl. }\end{array}$ & $\begin{array}{l}\text { Papel bond, } \\
\text { cartulinas, } \\
\text { crayolas, } \\
\text { plumones, } \\
\text { pinturas, } \\
\text { pegamento, } \\
\text { tijeras, recortes. }\end{array}$ \\
\hline
\end{tabular}

Fuente: Elaboración propia a partir de la información proporcionada por el DOC4.

Lo realizado por el maestro en el salón de clases durante la primera sesión de trabajo transcurrió de acuerdo con lo que planeó, abordó los aprendizajes esperados marcados por el currículum mediante actividades con las que movilizó elementos de la cultura indígena. Es importante que los docentes de esta modalidad educativa consideren que: "[...] la planeación y su puesta en práctica necesitarán favorecer el bilingüismo oral y escrito de cada estudiante, considerando que la escritura formal de las lenguas indígenas no está establecida aún para todas" (SEP, 2017, p. 43).

Para los docentes de estas escuelas no existe un referente programático rígido o prescriptivo para planear la enseñanza. En ambas instituciones me expresaron que los únicos documentos normativos en los que se basan para planear son los Parámetros Curriculares de la Asignatura Lengua Indígena (DGEI-SEP, 2008), así como los Aprendizajes Clave para la Educación Integral de Educación Primaria 
Indígena (SEP, 2017), esto se debe a que no cuentan con otros documentos u orientaciones pedagógicas.

Un escenario positivo de esta condición es que cuentan con una libertad relativa para preparar sus clases y hacerlas corresponder con las características y necesidades de los alumnos, las actividades son ajustadas a los recursos disponibles y a su contexto cultural. Sin embargo, la situación pudiera tornarse complicada en el caso de docentes que no tengan la experiencia suficiente para planear, y/o cuando desconocen a los alumnos y a la cultura del lugar. Por otro lado, sería ideal que a partir de las experiencias pedagógicas exitosas, fueran sistematizando y compartiendo con otros docentes de la misma modalidad educativa la organización y planeación de sus prácticas, no huelga decir también, que sería de mucha ayuda contar con más recursos materiales de la Secretaria de Educación Pública (SEP), de la Dirección General de Educación Indígena (DGEI), y de la Coordinación General de Educación Intercultural Bilingüe (CGEIB) para enriquecer el proceso de enseñanzaaprendizaje.

\section{Contenidos}

En los PD desarrollados en ambas escuelas, los maestros relacionan contenidos curriculares con saberes tradicionales y con el uso de la lengua indígena como vehículo de comunicación o expresión de lo aprendido o suscitado en el proceso de enseñanza-aprendizaje. La intraculturalidad puesta en práctica por medio de la enseñanza de saberes tradicionales, es una parte central de los proyectos de las escuelas indígenas, tiene como finalidad que los miembros de la comunidad educativa reconozcan y valoren las expresiones significativas de su cultura, la cual, no solo dota de identidad a las personas, sino también a su entorno, a la interacción que realizan con la naturaleza y a su historia (Riess, 2013).

Al hacer que los docentes miren hacia el interior de su propia cultura, la intraculturalidad promueve su valoración y reafirma la identidad. La intraculturalidad no es un concepto frecuente en el discurso educativo de los docentes, pero indudablemente, se trata de una herramienta epistemológica importante para el ejercicio de una educación intercultural, por lo tanto, es necesario que se capacite de manera sistemática en este tema a los maestros de educación indígena.

Como parte de las actividades del proyecto de "Herbolaria" desarrollado en la ESC2, la DOC2, encargada de primer y segundo grado, propuso que sus alumnos realizaran un álbum o compendio de plantas medicinales que les sirviera de apoyo en su exposición final, para ello, trabajó con los siguientes contenidos curriculares correspondientes al primer ciclo de educación primaria:

- Elaborar álbumes o compendios con palabras u oraciones cortas.

- Leer y escribir en el sistema de escritura de su lengua.

- Aporta preguntas, saberes y experiencias sobre un tema de investigación.

En la sesión destinada para este trabajo, sucedió lo siguiente: 
La maestra inició explicando a los alumnos que realizarían un álbum de plantas medicinales con la información recabada previamente con sus familiares y vecinos, puntualizó que lo irían complementando sesión a sesión y preguntó de manera abierta a todo el grupo:

DOC2 ¿Qué información encontraron sobre plantas medicinales?

Alejandro: Mi mamá me dijo que cuando tienes dolor de muela te debes poner un clavo de comer.

DOC2: ¿Un clavo de comer? ¡Se te van a caer los dientes con eso! Los clavos no se comen, eso es peligroso.

Hubo risas de otros alumnos. El comentario de la maestra fue a modo de provocación para que Alejandro expusiera de otra manera su respuesta y no creara confusión con sus compañeros.

Alejandro: ¡Un clavo de los que se usan para la comida, no de fierro!

DOC2: ¡Ah! Un clavo de especia; y eso, ¿cómo se usa?

Alejandro: Te lo pones en la muela que te duele para que se te quite el dolor.

DOC2: Muy bien, ¿qué otra planta medicinal investigaron?

Emilio: La higuerilla sirve para cuando te da calentura, te la ponen en la panza con manteca y te enredan un trapo, así nos hace mi abuelita.

Otros alumnos continuaron mencionando plantas y la maestra fue incitándolos a que formularan explicaciones preguntándoles qué propiedades tenían, si ya las habían utilizado, de qué manera, si las habían comido o bebido, a qué sabían, que olor tenían, si su uso era agradable o desagradable, donde se podían conseguir. La maestra realizó un listado en el pizarrón con el nombre de las plantas que los niños habían mencionado, algunos las llevaban escritas en su cuaderno y otros las habían memorizado, se pudo notar que eso no les representó alguna dificultad debido a que es una temática de su vida cotidiana. Posteriormente les repartió hojas blancas y les pidió que eligieran cinco plantas medicinales de la lista y las anotaran en las hojas, escribiendo su nombre tanto en español como en náhuatl, y, que, además, si las conocían debían dibujarlas. Una vez que los niños terminaron la actividad, la maestra recogió las hojas y las guardó en sus carpetas, aclarándoles que con las plantas que se fueran sumando, cada uno elaboraría su álbum de plantas medicinales que fueran de su interés (Diario de campo, 2020). 
Figura 1.

Trabajo de Emilio.

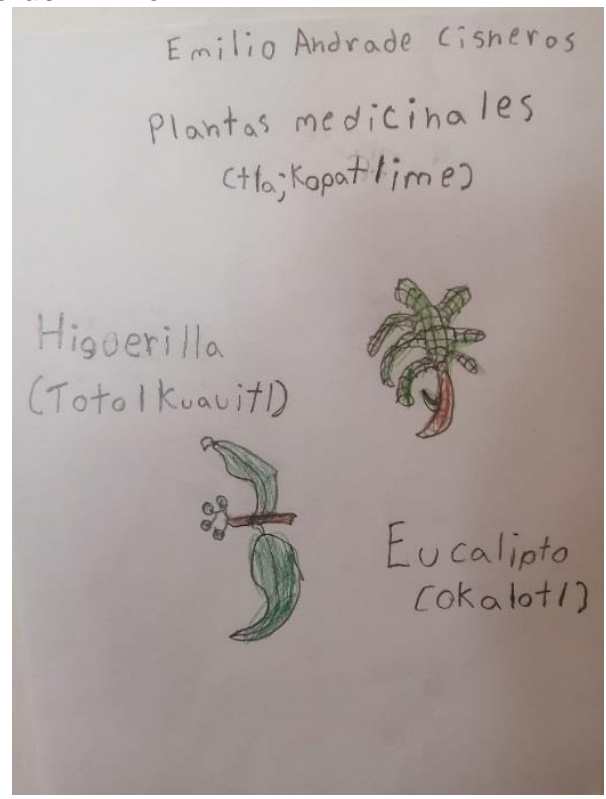

Fuente: Elaboración propia, enero, 2020.

\section{Significatividad en lo que aprenden}

Los contenidos curriculares adquieren significatividad cuando se relacionan con la cultura a la que el aprendiz pertenece, la finalidad de la intervención educativa es que los alumnos aprendan a pensar y a actuar sobre contenidos contextualizados y por ende significativos (Díaz Barriga y Hernández Rojas, 1997); por ello, en el parecer de los docentes, si los contenidos que se abordan no son significativos tampoco serán pertinentes.

Esto requiere que los docentes reflexionen sobre la historia y la cosmovisión propia, "entendida ésta como la manera en que el pueblo ha establecido relaciones y construidos conocimientos sobre el mundo que nos rodea, sobre su percepción del tiempo y del espacio, de la geografía, la naturaleza, la humanidad y el cosmos" (Riess, 2013, p. 20). Este ejercicio reflexivo sobre su propia cultura es determinante para que puedan dotar de significatividad a los contenidos curriculares, ya que la identificación con su cultura y los conocimientos que tienen sobre ella favorece la realización de una mejor contextualización de los contenidos programáticos. El DOC4 lo manifiesta de la siguiente manera:

Se necesita estar preparado como docente para hacer buenos materiales, hablar la lengua indígena, tener los conocimientos suficientes y adecuarlos a la comunidad, por ejemplo, el programa te indica que debes preparar galletas, 0 una pizza, pues entonces preparamos mejor una salsa de huevo, o de puerco, que es lo que tienen al alcance; en matemáticas a sacar cuanto mide una vara, cuantos metros tiene la tarea, etc., tienen que ser conocimientos prácticos para ellos, hacerlos significativos (DOC 4, comunicación personal, 2020). 
A través de la intraculturalidad se busca también que los alumnos valoren su cultura, esta también es otra condición para que los aprendizajes sean significativos, interesantes y pertinentes. Los maestros señalan que en su trabajo educativo por medio de PD buscan que los miembros de su comunidad educativa reconozcan y valoren elementos como:

El idioma, las tradiciones, la vestimenta regional, la gastronomía, la manera de cocinar, la forma de trabajo en el campo, las plantas medicinales, la elaboración de orfebrería, las formas en las que se organiza la comunidad, el respeto por la naturaleza, el respeto a la diversidad (DOC4, comunicación personal, 2020).

\section{Transversalidad en los aprendizajes}

Otra característica del trabajo por PD llevado a efecto en estas escuelas es la transversalidad en la enseñanza de los aprendizajes, a partir del tema principal se articulan una serie de contenidos programáticos que se abordan desde diferentes asignaturas, de esta manera, el aprendizaje adquiere un carácter holístico dado que los alumnos lo construyen desde distintas perspectivas, así lo explica la DOC3:

Con los proyectos hemos trabajado de manera transversal con distintas asignaturas, por ejemplo, con el proyecto del maíz, en el caso de mi grupo ( $1^{\circ}$ y $2^{\circ}$ Grado) trabajamos la lectoescritura en náhuatl y en español, trabajamos con leyendas y poesías cortas sobre el maíz, su historia desde un punto de vista sencillo, al nivel de los alumnos, vimos los ecosistemas en los que se siembra, el trabajo incluye varias materias. (DOC3, comunicación personal, 2020).

Esta forma de trabajo tiene sustento en el perfil que los Parámetros Curriculares de la Asignatura de Lengua Indígena (DGEI-SEP, 2008) les requiere de los docentes, entre los rasgos que deben considerar los maestros de educación indígena está el de "Organizar el tiempo escolar de manera flexible e integrar los contenidos de lengua indígena con los contenidos de otras asignaturas cuando sea pertinente hacerlo a fin de aprovechar al máximo los tiempos escolares" (p. 15), conviene agregar, que por tratarse de escuelas multigrado en las que los docentes atienden más de un grado, la transversalidad contribuye a la optimización de tiempo.

\section{Los conocimientos previos}

El trabajo por proyectos ha favorecido el trabajo con un enfoque constructivista, el flujo de los conocimientos en el proceso enseñanza-aprendizaje dejó de ser unidireccional de los maestros hacia los alumnos, bajo esta forma de trabajo todos los actores han experimentado la posibilidad de aportar experiencias y saberes al proceso educativo. Los docentes tienen claro que se trata de llevar a efecto una práctica educativa diferente a la concebida tradicionalmente, por ello, consideran de primer orden los conocimientos previos con los que cuentan sus alumnos, no solo porque resultan un referente que da cuenta de su situación académica, sino porque se han percatado, que los infantes también son capaces de hacer aportes valiosos al proceso educativo. Al respecto el DOC4 señaló: "Debemos ver también lo qué saben 
los niños, a veces pensamos que los niños no saben, pero en realidad ellos pueden aportar conocimientos, no debemos perder de vista lo que ya conocen" (DOC4, comunicación personal, 2020).

En la sesión en la que este maestro presentó el proyecto a sus alumnos, implícitamente exploró sus conocimientos previos con cuestionamientos que además tuvieron una función motivacional, ya que correspondían a un tema de su cultura:

DOC4: ¿De qué trata la celebración del día de muertos?

Miguel: Es para recibir a los muertitos porque cada año vienen a visitar a sus familiares.

Carmen: ¡Sí! Porque ese día vienen de visita

Adrián: Se celebra que los difuntos vienen a ver a su familia, y se comen lo que se les pone en la ofrenda para recibirlos porque está uno contento de que vienen.

DOC4: ¿Cómo es que se celebra?

José: En mi casa les ponemos la ofrenda, mi mamá hace chocolate y arroz para que coman.

Rogelio: Se les pone su ofrenda maestra, con todo lo que les gustaba cuando vivían porque el día de todos los santos vienen a comer y a visitar a sus familiares.

José: También se va al panteón a dejarles flores.

Soledad: ¡Mi abuelita hace mole, tiene unos guajolotes y ese día va a ser mole para los muertitos y para mi abuelito!

DOC4: Muy bien, pues lo que vamos a hacer es obtener la información necesaria para poner tlamanali (ofrenda) aquí en la escuela, ¿saben que es tlamanali?

Carmen, José, Jimena: ¡Ofrenda! (Diario de campo, ESC1, 2019).

\section{Materiales educativos}

Las escuelas cuentan principalmente con materiales educativos editados por la SEP, la DGEI y por la CGEIB. Estos materiales destinados a escuelas de la modalidad indígena están diseñados con un enfoque intercultural bilingüe para favorecer la enseñanza y el aprendizaje. Las escuelas también cuentan con materiales que han elaborado los mismos miembros de la comunidad educativa.

En estas escuelas cuentan con la biblioteca escolar, de la cual sobresale la colección semillas de palabras de la DGEI por ser la que más ejemplares aporta, dicha colección está integrada por cuentos, historias, anécdotas, poemas, refranes y adivinanzas escritas en once lenguas originarias, estas obras expresan las diferentes 
y particulares formas en que los pueblos perciben la vida, la cultura y la diversidad. Estos textos son útiles para trabajar la lengua en cada entidad, así como para fortalecer el trabajo de la multiculturalidad (PNUD, 2013). La biblioteca escolar se complementa con algunos cuentos editados por Consejo Nacional de Fomento Educativo (CONAFE), y otros que los docentes han conseguido por cuenta propia.

Por su parte, los alumnos solo cuentan con las guías-cuaderno de exploración del medio natural y sociocultural de los diferentes ciclos. Estas guías están destinadas a los pueblos originarios y afrodescendientes de México, tienen el propósito de que alumnas y alumnos profundicen en los conocimientos de sus ancestros, y se esperan que coadyuven al desarrollo de capacidades como las de escribir, hablar, escuchar y comprender en su lengua indígena (SEP, 2016).

La carencia de materiales educativos en las escuelas de educación indígena es una problemática generalizada, los maestros entrevistados coinciden que es necesario el suministro de materiales contextualizados para la enseñanza de la lengua, principalmente para los primeros grados en donde los alumnos adquieren y desarrollan la lectoescritura: "no hay materiales suficientes para trabajar, sobre todo en los primeros grados, hay libros que vienen en diferentes lenguas indígenas, pero para que los alumnos conozcan que existen otras lenguas, pero no para que trabajen con su propia lengua (DOC1, comunicación personal, 2020).

En el estado de Veracruz, las escuelas de la modalidad indígena son las más desfavorecidas en el rubro de materiales educativos, "no cuentan con laboratorios, espacios específicos para bibliotecas [...] hay escasez de materiales para educación física, desarrollo de artes, tratamiento de las ciencias naturales, entre otros" (Niembro y Mendoza, 2017, p. 102).

Para sortear estas carencias, los docentes elaboran materiales propios como tarjeteros o tendederos con letras del abecedario y palabras en náhuatl, los utilizan principalmente para la enseñanza de la lectoescritura. También elaboran compendios en náhuatl de cuentos breves, leyendas o canciones con los que abordan temas del ámbito de la literatura. Otros materiales que han elaborado son: "alfabetos en náhuatl, libros artesanales o cartoneros, memoramas, loterías, y en el caso de los proyectos los productos finales son álbumes y hasta videos (DOC4, Comunicación personal, 2020).

Un material elaborado con los PD ha sido el libro cartonero o artesanal, el cual, fue pensado para promover la lectura y la escritura entre la comunidad educativa a partir de textos situados basados en las prácticas sociales del lenguaje y en las formas particulares de interacción entre las personas, o de los grupos sociales a los que pertenecen (Olarte y Zacarías, 2014). Generalmente es elaborado al término de los proyectos por docentes, alumnos y padres de familia con insumos de la región, son escritos en lengua indígena, se editan y se integran a la biblioteca escolar para consulta. 


\section{Figura 2}

Elaboración del libro artesanal sobre la "celebración del día de muertos"

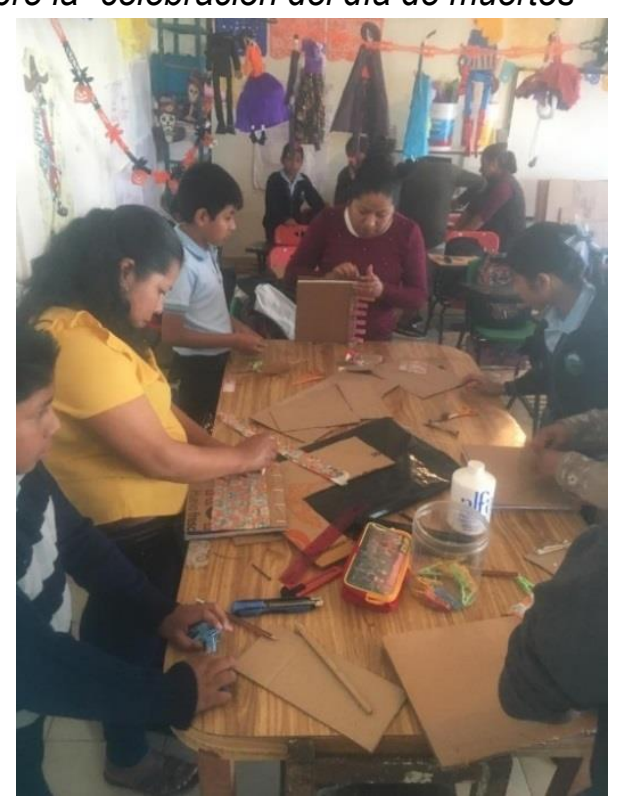

Fuente: Fotografía del archivo personal de la docente de sexto grado, octubre, 2019.

\section{Evaluación pertinente}

Para abordar este apartado presento a continuación un extracto del diario de campo sobre una actividad de evaluación que realizó la DOC3 en la ESC1 para conocer los avances de los alumnos en la ampliación de su vocabulario en náhuatl con palabras del tema del proyecto:

[...] a manera de repaso inició preguntando de manera abierta qué elementos se ponían en la ofrenda de muertos, los niños fueron contestando de manera desordenada en español, de modo que la maestra les pidió que levantaran la mano para que ella les diera el turno de la palabra, así lo hizo con alrededor de ocho alumnos, la mayoría de los participantes fueron de segundo grado. Posteriormente preguntó al grupo en general cómo se decían esas palabras que habían mencionado en lengua náhuatl, la participación de los alumnos no menguó, pero continuó en desorden, por lo que volvió a tomar la palabra y dio la instrucción de que debían levantar la mano para que ella llevara el orden de las participaciones, fue dando los turnos y los alumnos fueron contestando:

\section{Rosa: Agua-atl}

Luis Ángel: Incienso-kopalli

Fidencio: Sal-istatl

Lupita: Chocolate-chokolatl

Roberto: Tamales de frijol-etamalime

Concepción: Velas-tlawillime 
Posteriormente, la maestra colocó sobre el pizarrón una ofrenda dibujada en cartulinas que previamente había elaborado, sobre su escritorio colocó tiras de papel bond en las que se encontraban escritos en lengua náhuatl los elementos de la ofrenda del día de muertos y les explicó a los alumnos que pasaran a colocar una palabra sobre el dibujo que correspondiera, la maestra les fue pidiendo alumno por alumno que tomaran una palabra y la pegaran sobre el dibujo que correspondiera, solo Virginia tuvo problema con la palabra ixcopinalti (retrato), por lo que fue auxiliada por el grupo. A los alumnos de primer grado les pidió que respondieran de manera oral mientras señalaba los objetos, al final sólo José Manuel y Valentín no lograron contestar correctamente (Diario de campo, ESC1, 2019).

Figura 3

Actividad evaluativa del primer ciclo, ESC1.

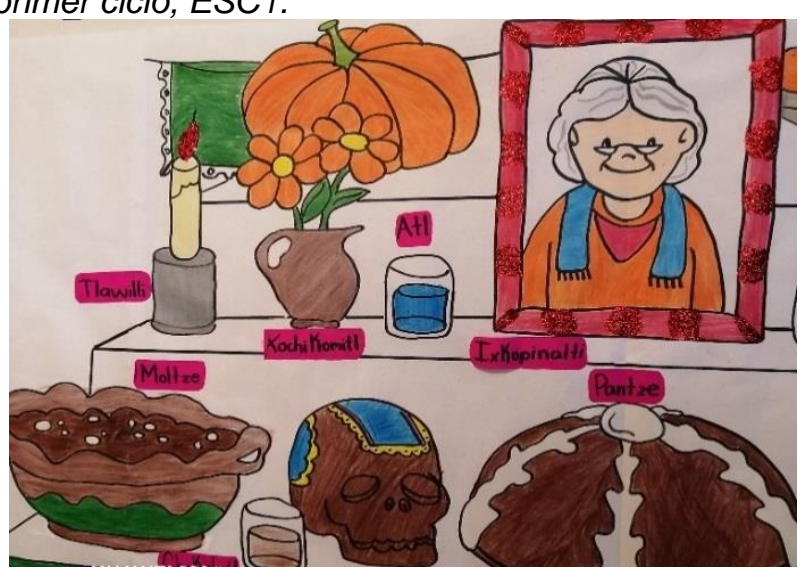

Fuente: Autoría propia, noviembre, 2019.

Para los entrevistados, la evaluación como requisito administrativo a través de instrumentos como el examen no tiene sentido, docentes de las dos escuelas coinciden con este punto: "No porque ya lo escriba en un examen quiere decir que el niño sepa, a lo mejor si sabe, pero no sabe cómo se hace, o no quiere participar en las actividades" (DOC1, comunicación personal, 2020).

Una finalidad de la evaluación es que los maestros puedan tomar decisiones para efectuar una enseñanza adaptada a la diversidad de sus alumnos, también les brinda la oportunidad de ofertar aprendizajes con sentido y valor funcional. El carácter pedagógico de la evaluación radica en que los docentes tengan la posibilidad de ajustar las circunstancias o condiciones en las que sus alumnos aprenden (Díaz Barriga y Hernández Rojas, 1997). Para estos maestros, es importante que sus alumnos demuestren sus aprendizajes adquiridos de forma observable al realizar determinados procesos, así como de manera actitudinal ante los demás miembros de la comunidad educativa, para tal efecto, realizan una evaluación por competencias. La DOC3 expresa su idea de evaluación:

[...]Con hechos, con procedimientos, ya vimos que no solo es importante el conocimiento, los alumnos deben demostrar que están aprendiendo cosas que a ellos les sirven, en su casa, en el respeto a los demás, o también con la 
naturaleza, a no tener malos comportamientos con sus compañeros (DOC3, comunicación personal, 2020).

EI DOC4, señala de manera concreta los rasgos que consideró cuando evaluó el proyecto de la celebración del día de muertos:

Los alumnos deben investigar, de compartir con sus compañeros lo que saben, deben participar, que, si se monta la ofrenda, por ejemplo, ellos deben saber cómo va y deben ayudar a ponerla [...] deben exponer lo que aprendieron con los demás compañeros y con la comunidad, deben hacerlo también en su lengua materna, ahí es donde te das cuenta si aprendieron (DOC4, comunicación personal, 2020).

Para este tipo de evaluación todos los dominios de aprendizaje son importantes, es decir, importan los conocimientos, las habilidades o desempeños, así como las actitudes de los estudiantes; la evaluación por competencias implica que los docentes diseñen instrumentos mediante los cuales los alumnos demuestren con ejecuciones que pueden realizar las tareas que demanda lo aprendido (García, s/f, p. 2).

Los instrumentos de los que pueden valerse los maestros son "entrevistas, exposiciones, producción de textos, proyectos, observaciones en situaciones espontáneas o planificadas, o instrumentos como rúbricas y portafolios de evidencias" (SEP, 2017, p. 43). Durante la implementación de los proyectos escolares, los docentes utilizan rúbricas, listas de cotejo, carpetas de evaluación, entre otros. Por ejemplo, en la sesión de clausura del proyecto de la celebración de día de muertos, los maestros a través de diferentes instrumentos registraron el desempeño de sus alumnos, durante la jornada, cada grupo expuso ante la comunidad educativa las actividades realizadas. Como actividad general montaron un altar de muertos, y con la ayuda de una persona socialmente reconocida por sus conocimientos en el tema, se realizó una explicación detallada de la tradición y del significado del altar, mientras los alumnos más avanzados asistían la exposición, otros tomaban nota de la información. La jornada culminó cuando retiraron el altar y llevaron a cabo una convivencia donde compartieron los alimentos que habían sido colocados en la ofrenda.

Seguramente existen áreas de oportunidad en la práctica educativa de los docentes de estas instituciones que pueden mejorarse, pero el trabajo que realizan deja constancia de su ímpetu por ofertar una educación pertinente para mejorar las condiciones de vida reales de sus alumnos, de prácticas educativas que favorecen la conservación del medio ambiente, el respeto por otras culturas, y la reafirmación de su identidad cultural. 


\section{Figura 4}

Presentación del altar del día de muertos en ESC1

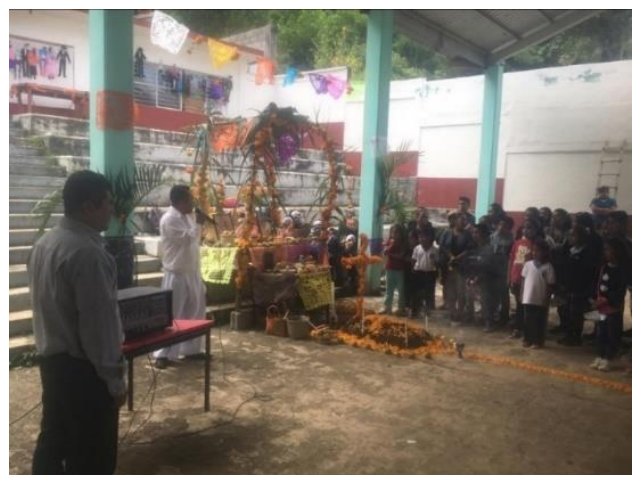

Fuente: Foto del archivo personal del DIR1, noviembre, 2019.

\section{Conclusiones}

A pesar de los esfuerzos para construir un currículum pertinente, la educación básica en México aún mantiene un carácter monocultural y monolingüe debido a que en términos reales no se considera a la diversidad como un elemento constitutivo de los contenidos nacionales (SEP-CGEIB, 2014). Por esa razón, una educación pertinente se convierte en una necesidad prioritaria para sociedades como la indígena, y un desafío para el sistema educativo.

Ante este escenario, los PD trabajados por las comunidades educativas de estas dos escuelas, indudablemente contribuyen a una educación cultural y lingüísticamente pertinente. La estrategia ha convertido a las escuelas en agentes institucionales importantes para revitalizar y preservar la lengua y la cultura nahua. Es de resaltar la labor docente en esta tarea, ya que, a pesar de recibir una capacitación limitada al respecto, los maestros crean y adaptan estrategias que implementan con pocos recursos materiales y educativos.

Con los PD, el proceso educativo ha trascendido el ámbito escolar, se han incorporado elementos como los saberes tradicionales generalmente asociados a la informalidad educativa, al mismo tiempo que actores comunitarios realizan aportes al proceso de aprendizaje, que, al conjuntarse con las acciones de los maestros, han impactado favorablemente en diferentes dimensiones de las comunidades.

Es primordial que las autoridades educativas asuman el compromiso de llevar a efecto acciones estratégicas para lograr que la educación que se oferta a las comunidades indígenas sea pertinente, para ello, se requiere que atiendan los problemas básicos de infraestructura, recursos humanos y educativos en las escuelas del subsistema indígena. 
Aunque se ha conseguido que el currículum se interculturalice en cierto grado, se requiere de mayor libertad y flexibilidad para que las comunidades educativas, en conjunto, planifiquen y conduzcan sus procesos educativos, superando la función integradora que tradicionalmente ha tenido la educación indígena.

Por último, si se pretende que los docentes consoliden prácticas educativas pertinentes que mejoren la educación ofertada a la población indígena, debe emprenderse una estrategia integral de capacitación sistemática con la que se logre transformar cualitativamente la realidad de las comunidades sin que pierdan su identidad y su legado cultural.

\section{Referencias}

Arias. L. (2017). El aprendizaje por proyectos: una experiencia pedagógica para la construcción de espacios de aprendizaje dentro y fuera del aula. Revista Ensayos Pedagógicos, XII (1), 51-68. https://bit.ly/3bGTJ7e

AVELI. (2010). Catálogo de lenguas indígenas y sus variantes lingüísticas del estado de Veracruz. Academia Veracruzana de las lenguas indígenas. https://bit.ly/3kraxDi

CONEVAL. (2015). Consulta dinámica de resultados de pobreza a nivel municipio 2010 y 2015. CONEVAL https://bit.ly/3uApilw

DGEI (2008). Parámetros Curriculares de la Asignatura de Lengua Indígena. DGEISEP.

Díaz-Barriga F. y Hernández G. (1997). Estrategias docentes para un aprendizaje significativo: una interpretación constructivista. Mc Graw Hill.

Dietz, G. (2017). La construcción e interpretación de datos etnográficos. En DíazBarriga y Domínguez (Coords.), La interpretación: un reto a la investigación educativa (229-262). Newton. Innovación y tecnología educativa.

García, J. (s/f). La evaluación por competencias. [Curso-Taller] Educando para una Formación Integral. México. ITESM.

Hevia, R.; Hirmas, C. y Peñafiel, S. (2002). Pertinencia cultural para mejorar los aprendizajes. Tarea Revista de Educación y Cultura, núm. 53, 32-40. https://bit.ly/3aWWLF7

INEGI. (2010). Censo Nacional de Población y Vivienda 2010. INEGI.

Jiménez, Y. y Mendoza, G. (2012). Evaluación integral, participativa y de política pública en educación indígena desarrollada en las entidades federativas. SEPSEV. https://bit.ly/3rcpqfr

Mendoza, G. y Niembro, C. (2017). La educación indígena en Veracruz: diagnóstico y recomendaciones para la política educativa. Universidad Veracruzana.

Muñoz, H. (1999). Política pública y educación indígena escolarizada en México. Cadernos Cedes, núm.49, 39-61. https://bit.ly/3bKM2Nu 
Olarte Tiburcio, E. y Zacarías Candelario, J. (2014). Libro cartonero: una alternativa para la integración a la cultura escrita en lengua indígena. Correo del Maestro, (223). https://bit.ly/3bGTTLS

Petrovic, M. (2017). El estatus del náhuatl como lengua minoritaria. Beoiberística, 1(1), 43-62. https://bit.ly/2O2ZMeb

PNUD. (2013). Evaluación de Proceso de cuatro Programas y Estrategias de Educación indígena. SEP-ONU.

Riess, B. (2013). Intraculturalidad y materiales didácticos. Metodología para la recuperación de saberes. CGEIB-SEP. https://bit.ly/3uE4pwf

Salazar, B.; Saldívar, A.; Limón, F.; Estrada, E.; Fernández, E. (2015). Pertinencia cultural y evaluación educativa en los Altos de Chiapas. Experiencias de estudiantes y profesores sobre la prueba ENLACE. Revista Latinoamericana de Estudios Educativos, XLV (3), 81-117. https://bit.ly/3bBBaRK

SEFIPLAN (2018). Sistema de información municipal. Cuadernillos municipales. Gobierno del Estado de Veracruz. https://bit.ly/3syQS7f

SEP. (2016). Guías-cuaderno de exploración del medio natural y sociocultural. SEP.

SEP. (2017). Aprendizajes clave para la educación integral. Educación primaria indígena. Atención a la diversidad y Lenguaje y Comunicación. SEP.

SEP-CGEIB. (2014). El enfoque intercultural en educación. Orientaciones didácticas para maestros de primaria. SEP-CGEIB.

SEP-DGEI. (1990). Diagnóstico para la modernización de la educación indígena. SEP-DGEI.

UNESCO. 2007. Educación de calidad para todos: un asunto de derechos humanos. Documento de discusión sobre políticas educativas en el marco de la II Reunión Intergubernamental del Proyecto Regional de Educación para América Latina y el Caribe (EPT/PRELAC). UNESCO- Oficina Regional de Educación para América Latina y el Caribe. https://bit.ly/2ZVsGji

Vázquez López, José Jaime, \& Valenzuela Ojeda, Gloria Angélica. (2013). Del análisis institucional a la etnografía institucionalista: experiencias y conceptos franceses. Entrevista a Patrick Boumard. Revista electrónica de investigación educativa, 15(1), 1-17. https://bit.ly/3r7q2mc 
